\title{
Frequent MAGE Mutations in Human Melanoma
}

\author{
Otavia L. Caballero ${ }^{1 * 9 \times}$, Qi Zhao ${ }^{29}$, Donata Rimoldi ${ }^{3}$, Brian J. Stevenson ${ }^{3}$, Suzanne Svobodová ${ }^{4}$ Sylvie \\ Devalle ${ }^{1}$, Ute F. Röhrig ${ }^{3}$, Anna Pagotto ${ }^{5}$, Olivier Michielin ${ }^{3}$, Daniel Speiser ${ }^{3}$, Jedd D. Wolchok ${ }^{1,6}$, Cailian \\ Liu $^{6}$, Tanja Pejovic ${ }^{7}$, Kunle Odunsi ${ }^{8}$, Francis Brasseur ${ }^{9}$, Benoit J. Van den Eynde ${ }^{9}$, Lloyd J. Old ${ }^{1}$, Xin Lu ${ }^{5}$, \\ Jonathan Cebon ${ }^{4}$, Robert L. Strausberg ${ }^{10}$, Andrew J. Simpson ${ }^{10}$
}

1 Ludwig Institute for Cancer Research Ltd, New York Branch at Memorial Sloan-Kettering Cancer Center, New York, New York, United States of America, 2 J. Craig Venter Institute, Rockville, Maryland, United States of America, $\mathbf{3}$ Ludwig Institute for Cancer Research Ltd, Lausanne Branch, Lausanne, Switzerland, $\mathbf{4}$ Ludwig Institute for Cancer Research Ltd, Melbourne Centre for Clinical Sciences, Austin Health, Heidelberg, Victoria, Australia, 5 Nuffield Department of Clinical Medicine, University of Oxford, Ludwig Institute for Cancer Research Ltd, Oxford Branch, Headington, Oxford, United Kingdom, 6 Department of Medicine and Ludwig Center for Cancer Immunotherapy, Memorial Sloan-Kettering Cancer Center, New York, New York, United States of America, 7 Division of Gynecologic Oncology and the Knight Cancer Institute, Oregon Health \& Science University, Portland, Oregon, United States of America, 8 Department of Gynecological Oncology and Center for Immunotherapy, Roswell Park Cancer Institute, Buffalo, New York, United States of America, 9 Ludwig Institute for Cancer Research Ltd, Brussels Branch, Université catholique de Louvain, Brussels, Belgium, 10 Ludwig Institute for Cancer Research Ltd, New York, New York, United States of America

\begin{abstract}
Background: Cancer/testis (CT) genes are expressed only in the germ line and certain tumors and are most frequently located on the X-chromosome (the CT-X genes). Amongst the best studied CT-X genes are those encoding several MAGE protein families. The function of MAGE proteins is not well understood, but several have been shown to potentially influence the tumorigenic phenotype.

Methodology/Principal Findings: We undertook a mutational analysis of coding regions of four CT-X MAGE genes, MAGEA1, MAGEA4, MAGEC1, MAGEC2 and the ubiquitously expressed MAGEE1 in human melanoma samples. We first examined cell lines established from tumors and matching blood samples from 27 melanoma patients. We found that melanoma cell lines from $37 \%$ of patients contained at least one mutated MAGE gene. The frequency of mutations in the coding regions of individual MAGE genes varied from $3.7 \%$ for MAGEA1 and MAGEA4 to $14.8 \%$ for MAGEC2. We also examined 111 fresh melanoma samples collected from 86 patients. In this case, samples from $32 \%$ of the patients exhibited mutations in one or more MAGE genes with the frequency of mutations in individual MAGE genes ranging from $6 \%$ in MAGEA1 to $16 \%$ in MAGEC1.
\end{abstract}

Significance: These results demonstrate for the first time that the MAGE gene family is frequently mutated in melanoma.

Citation: Caballero OL, Zhao Q, Rimoldi D, Stevenson BJ, Svobodová S, et al. (2010) Frequent MAGE Mutations in Human Melanoma. PLoS ONE 5(9): e12773. doi:10.1371/journal.pone.0012773

Editor: Patrick Tan, Duke-National University of Singapore Graduate Medical School, Singapore

Received July 12, 2010; Accepted August 17, 2010; Published September 16, 2010

Copyright: (C) 2010 Caballero et al. This is an open-access article distributed under the terms of the Creative Commons Attribution License, which permits unrestricted use, distribution, and reproduction in any medium, provided the original author and source are credited.

Funding: This work was conducted as part of the Hilton-Ludwig Cancer Metastasis Initiative, funded by the Conrad N. Hilton Foundation and the Ludwig Institute for Cancer Research. The funders had no role in study design, data collection and analysis, decision to publish, or preparation of the manuscript.

Competing Interests: The authors have declared that no competing interests exist.

*E-mail: ocaball1@jhmi.edu

9 These authors contributed equally to this work.

a Current address: Department of Neurosurgery, Johns Hopkins University School of Medicine, Baltimore, Maryland, United States of America

\section{Introduction}

Cancer/testis (CT) genes are expressed primarily in the germ line but are also active in a number of human tumors including those of the lung, breast, ovary and skin [1]. Amongst the CT-genes is a subset with very tight transcriptional regulation that is specifically expressed in spermatogonia, completely undetectable in somatic tissues and encoded on the $\mathrm{X}$-chromosome [2]. The proteins derived from these CT-X genes are significantly immunogenic when aberrantly expressed in human tumors and are being widely studied in the context of therapeutic cancer vaccines [3,4]. Currently, two phase III trials are being undertaken with a vaccine containing the CT-X protein MAGEA3 as an adjuvant therapy for non-small cell lung cancer and melanoma [5].

Due to their strong up regulation in tumors, it has been widely speculated that the CT-X genes might play a role in the tumorigenic process. This has been difficult to prove, however, as their function remains obscure. Nevertheless a number of in vitro studies, focused on the MAGE proteins, have found evidence that they can interfere with p53 mediated apoptosis and promote cell proliferation $[6,7,8,9,10]$. In addition, a number of studies have found CT-X expression to be linked with both more advanced and more aggressive tumors $[11,12,13]$. To complicate this scenario, however, there have also been observations that link the expression of individual MAGE genes with a better prognosis and longer survival $[14,15,16]$.

Recently, it has begun to be possible to undertake genome-wide investigations of somatic mutations in human tumors $[17,18,19]$. Within the published data, we identified reports of missense mutations in the CT-X antigen genes MAGEA1, MAGEA4, $M A G E C 1, M A G E C 2$, as well as the genes for the ubiquitously expressed MAGEE1 (also encoded on the X chromosome) in breast 
and brain tumors [17,18]. Although the frequency of these mutations is low, we reasoned that their mutation might not be simply due to chance as none were observed to be mutated in colon or pancreatic tumors although the same genes were sequenced in similar numbers of tumors $[17,19]$. Furthermore, MAGEE1 was mutated sufficiently frequently to be classified as a candidate cancer gene (CAN-gene) in breast cancer and thus potentially a driver of tumorigenesis [17].

Since greater knowledge of somatic $M A G E$ mutations in human tumors might cast further light on their potential role in tumorigenesis, as well as provide important information relevant to the use of MAGE proteins in cancer vaccines, we have undertaken a systematic mutational analysis of the coding regions of the five MAGE genes in which mutations were reported (MAGEA1, MAGEA4, MAGEC1, MAGEC2, MAGEE1). For this study we used human melanoma and ovarian samples, two tumor types with frequent CT-X expression. Our results reveal that one or more of these genes is mutated in around $35 \%$ of melanomas with some tumors exhibiting multiple mutations in these genes. On the other hand we found no mutations of these genes in ovarian tumors. Further investigations will be required to determine whether these mutations are drivers or passengers of tumorigenesis.

\section{Materials and Methods}

\section{Sequence analysis of the melanoma samples}

Tumor and matching blood samples from 27 melanoma cancer patients were collected at Lausanne University Hospital (CHUV), Switzerland. Cell lines were established from these samples at the Ludwig Institute of Cancer Research, from fresh surgery samples using mechanical or a combination of mechanical and enzymatic dissociation. All cell lines were derived from cutaneous melanomas, except for T1257A and B (mucosal melanoma). They were all from tumor metastases, except for LAU-Me300 and LAUT1257A, which were from primary tumors. The following pairs of cell lines were established from the same patients: LAUMe260.LN and LAU-T149D (patient 149, 7 years apart); LAUMe275 and LAU-T50B (patient 50, 12 years apart); LAU-Me 261 and LAU-T42B (patient 42, 3 years apart); LAU-Me305 and LAU-Me317.M2 (patient 233, 6 months apart); LAU-T1257A and $\mathrm{C}$ (patient 1257, primary tumor and synchronous metastasis, respectively); LAU-T1262 A and B (patient 1262, synchronous metastases); LAU-T1255A/B are two independent lines from a large tumor. Established cultures were confirmed to be from human melanoma by flow cytometric analysis with antibodies against the high molecular weight melanoma- associated antigen and MHC class I molecules. Additional phenotyping was performed by flow cytometry, Western blotting and RT/PCR to assess expression of melanoma/melanocytic antigens (e.g. MART1, tyrosinase, cancer/testis genes). Cell lines were routinely tested and found negative for mycoplasma. Cells were periodically checked for morphology and expression of selected antigens by RT/PGR.

In addition, fresh tumor and blood samples were collected from 86 patients attending the Melanoma Clinic at Austin Health, Melbourne, Australia (Table S2). A written informed consent was obtained from all participating subjects. This study was approved by the Ethics Committee for Clinical Research from the University of Lausanne, Switzerland, by the Human Research Ethics Committee, Research Ethics Unit, Austin Hospital, Australia and by the Ethics Committee, J. Craig Venter Institute.

Genomic DNA was extracted using a Qiagen kit following a standard protocol. Targeted sequencing was carried out with a fully automated and high-throughput production pipeline that is based on polymerase chain reaction (PCR) amplification of genomic DNA followed by traditional Sanger sequencing chemistry as previously described [20]. Primer sequences are listed in Table S3. Mutational analysis was done by comparing the sequence traces between tumors and their matching blood samples. Each somatic mutation call had to be supported by both forward and reverse traces of each amplicon and was manually verified.

\section{Cloning of and sequencing analysis of mutated MAGEA1 in LAU-Me190}

PCR was undertaken with High Fidelity Taq polymerase (Invitrogen, Carlsbad, CA) plus 10 pmol of each of the following primers in $25 \mu \mathrm{l}$ to amplify the region containing the sequence variation in the tumor corresponding to the LAU-Me190 cell line: Forward 5'-AGAAAACCAACCAAATCAGCCA-3' and Reverse 5'-TCATGTCTCTTGAGCAGAGGAGTCT-3'. The amplification consisted of 35 cycles of a denaturation step at $94^{\circ} \mathrm{C}$ for $30 \mathrm{~s}$, followed by $30 \mathrm{~s}$ at $55^{\circ} \mathrm{C}$ and extension at $68^{\circ} \mathrm{C}$ for $30 \mathrm{~s}$ followed by a final 7 -min extension. PCR products were loaded onto $1.5 \%$ agarose gel, stained with ethidium bromide and visualized by UV illumination. The predicted size of the MAGEAl PCR product was $340 \mathrm{bp}$. The PCR product was recovered and purified after agarose gel electrophoresis using a QIAquick Gel Extraction Kit (Qiagen, Valencia, CA). Cloning in $\mathrm{pcDNA}^{\mathrm{TM}_{3}}$.1/V5-His was performed at room temperature for 30 minutes in a total volume of $6 \mu \mathrm{l}$ using the pcDNA ${ }^{\mathrm{TM}_{3.1}} / \mathrm{V} 5-\mathrm{His} \mathrm{TOPO}^{\circledR} \mathrm{TA}$ Expression Kit (Invitrogen, Carlsbad, CA). Transformation was performed into chemically competent One Shot ${ }^{\circledR}$ TOP10 E. coli (Invitrogen, Carlsbad, CA) that were plated in LB plates containing $100 \mu \mathrm{g} / \mathrm{ml}$ ampicillin and incubated overnight at $37^{\circ} \mathrm{C}$. Fifteen colonies were picked and grown overnight in LB medium containing $100 \mu \mathrm{g} / \mathrm{ml}$ ampicillin. Plasmids were isolated with Wizard ${ }^{\circledR}$ Plus SV Minipreps DNA Purification System (Promega Madison, WI). DNA was submitted to Sanger sequencing using the T7 promoter primer.

\section{Results and Discussion}

The entirety of MAGEA1, MAGEA4 and MAGEC2 genes and at least $70 \%$ of MAGEC1 and MAGEE1 could be specifically PCR amplified thus permitting detection of somatic mutations by conventional Sanger sequencing. Some regions of MAGEC1 and MAGEE1 could not be covered by PCR amplicons due to very high GC content or repetitive sequences.

To avoid the complication of contaminating normal tissues in fresh tumor samples, we first undertook a Discovery Screen for MAGE mutations using melanoma cell lines and corresponding EBV transformed leukocytes from 27 patients treated at the University Hospital in Lausanne, Switzerland (CHUV). We detected a total of 15 somatic MAGE coding region mutations in these cell lines, with at least one mutation in each of the five genes examined (Table 1). Two other genes were also sequenced in these samples, PRAME, on chromosome 22 and DDX53 on the $\mathrm{X}$ chromosome, and served as negative controls as no mutations were found. As a positive control, the expected mutation frequencies of the major cancer genes TP53 and BRAF were found in these cell lines (Table $\mathrm{S} 1$ ).

Overall, cell lines from 10 of the 27 patients exhibited MAGE mutations $(37 \%)$. From five of the patients where MAGE mutations were found, fresh tumor tissue was also available. In four of these we were also able to identify the mutation found in the cell line in the fresh tissue. The exception was the tumor 
Table 1. Somatic mutations identified in MAGE genes in the discovery set.

\begin{tabular}{|c|c|c|c|c|c|}
\hline Gene & Sample name & Amino acid & Codon change & TP53 status & BRAF status \\
\hline MAGEA1 & LAU-Me190 & S33F & $\mathrm{TCC}>\mathrm{TTC}$ & WT & V600E \\
\hline MAGEA1 & LAU-Me190 & L129L & $\mathrm{CTG}>\mathrm{CTA}$ & WT & V600E \\
\hline MAGEA4 & LAU-Me190 & E34K & $\mathrm{GAG}>\mathrm{AAG}$ & WT & V600E \\
\hline MAGEC1 & LAU-Me190 & E877K & $\mathrm{GAG}>\mathrm{AAG}$ & WT & V600E \\
\hline MAGEC1 & LAU-Me190 & F144F & $\pi \mathrm{TC}>\pi T$ & WT & V600E \\
\hline MAGEC1 & LAU-Me243 & P756S & $\mathrm{CCC}>\mathrm{TCC}$ & WT & WT \\
\hline MAGEC1 & LAU-Me200 & G769R & GGG $>$ AGG & S241F & WT \\
\hline MAGEC2 & LAU-Me275 & S110N & $A G C>A A C$ & WT & V600E \\
\hline MAGEC2 & LAU-Me243 & $\mathrm{R} 271 \mathrm{R}$ & $A G G>A G A$ & WT & WT \\
\hline MAGEC2 & LAU-Me300 & S111L & $\mathrm{TCA}>\mathrm{TTA}$ & WT & V600E \\
\hline MAGEC2 & LAU-Me280.R.LN & P295L & $\mathrm{CCA}>\mathrm{CTA}$ & P278S & G593S; L597R \\
\hline MAGEC2 & LAU-Me261 & S51F & $\mathrm{TCC}>\mathrm{TTC}$ & S241F & WT \\
\hline MAGEE1 & LAU-Me290 & P324S & $\mathrm{CCT}>\mathrm{TCT}$ & WT & WT \\
\hline MAGEE1 & LAU-T441A & P568L & $\mathrm{CCC}>\mathrm{CTC}$ & WT & WT \\
\hline MAGEE1 & LAU-Me281 & Т330Т & $A C C>A C G$ & WT & V600E \\
\hline
\end{tabular}

doi:10.1371/journal.pone.0012773.t001

matching LAU-Me190 cells (five different mutations were found in the latter). One explanation for this could be tumor heterogeneity, which would render mutations present in small subset of cells undetectable by the sequencing technology used. To investigate this possibility, we selected one mutation, S33F in MAGEA1, for further study. We amplified the mutated region from the tumor tissue and cloned the amplification products into a plasmid and sequenced the clones. Two of 15 clones were found to contain the mutation. Thus for this mutation we were able to confirm the mutation in the original tissue in a subset of alleles.

Two of the mutations detected occurred in cell lines from patients for which additional autologous melanoma lines established from separate metastases were available. In one case, the lines (LAU-Me275 and LAU-T50B) were established twelve years apart and in the second (cell lines LAU-Me261 and LAU-T42B), the lines were established three years apart. We tested these additional lines for the presence of the mutations. In both cases, the mutations were found in the paired asynchronous cell lines.

Based on our finding of MAGE mutations in the melanoma cell line samples, a Validation Screen was undertaken in which we sequenced the same $M A G E$ genes in 111 fresh tumor samples collected from 86 melanoma patients who had undergone surgical intervention at the Melanoma Clinic at Austin Health, Melbourne, Australia (Table 2). In addition, we sequenced the same genes in 33 samples from ovarian tumors collected at Roswell Park Cancer Center, Buffalo, New York. We identified additional somatic coding region mutations for each of the genes in the melanoma samples. Overall, $32 \%$ of the melanoma patients had a mutation in at least one of the genes sequenced. The frequencies of patients with missense or nonsense mutations for the individual genes were $5.8 \%, 11.6 \%, 15.1 \%$ and $7.0 \%$ and $7.0 \%$ for MAGEA1, MAGEA4, MAGEC1, MAGEC2 and MAGEE1 respectively. More than one sample was available from six of the patients. In all cases where a $M A G E$ mutation was found in one sample, it was also found to be present in the other samples from the same patient. We observed coding region mutation frequencies of $47.8 \%$ and $20.4 \%$ respectively for BRAF and TP53 in the samples in the Validation Screen (Table S2). These frequencies are both consistent with those reported by others for these genes in melanoma arguing that our findings are representative. In contrast to the melanoma samples, no MAGE mutations were identified in any of the ovarian samples sequenced although a mutation frequency of $31 \%$ was found for TP53 (Table S4). This frequency is consistent with the findings of others [21] suggesting that the samples were sufficiently enriched for tumor derived cells to permit the detection of $M A G E$ mutations were they to be present. The apparent lack of mutations in the ovarian samples studied points to a distinct tumorigenic pathway for the ovarian tumors and melanoma where MAGE genes may play different roles.

In addition to generating new mutation data, we also combed the publically available databases and found a small number of additional mutations in lung tumors, glioblastoma, breast tumors and melanoma in MAGE genes [17,18,19,22] (Table 3). On the other hand, some tumor types known to express CT-X genes, such as colon, bladder and ovarian (as confirmed here), have no recorded mutations to date.

The recent sequencing of a melanoma genome has revealed a mutation spectrum reflective of the mutagenic impact of ultraviolet light [23]. This pattern can be clearly observed in this study in that $\mathrm{C}>\mathrm{T}-\mathrm{G}>\mathrm{A}$ alterations represent almost $89 \%$ of the $M A G E$ mutations found (Table S5), with over $90 \%$ of these occurring at dipyrimidine sites. This UV mutation signature pattern was also observed for TP53 in the melanoma samples analyzed here. It was not, however, observed in the MAGE mutations found in other tumor types.

Overall, the non-synonymous to synonymous ratio of the $M A G E$ mutations was found to be 2.45:1. This is not different from the ratio that would be expected to occur by chance. However, this low non-synonymous:synonymous ratio is largely due to a very high proportion of synonymous mutations in MAGEC1 and MAGEC2 (14 of 20). MAGEA1, MAGEA4, and MAGEE1 have non-synonymous to synonymous ratios of $6: 1,5: 1$ and 2.66:1 respectively. Moreover, all the mutations in the two MAGEA genes reported in the databases are non-synonymous (Table 1). Thus, MAGEA mutations might be drivers of tumorigenesis, as has previously been postulated for MAGEE1. Due to their distribution throughout the genes studied (Figure 1), we speculate that the $M A G E$ mutations we have identified are more likely to be 
Table 2. Somatic mutations identified in MAGE genes in the validation set.

\begin{tabular}{|c|c|c|c|c|c|}
\hline Gene & Sample name & Amino acid & Codon change & TP53 status & BRAF status \\
\hline MAGEA1 & 04-007 & S296P & $\mathrm{TCC}>\mathrm{CCC}$ & WT & V600E \\
\hline MAGEA1 & 7552 & L271F & $\mathrm{CTC}>\mathrm{TTC}$ & WT & V600E \\
\hline MAGEA1 & 4198 & E217K & $\mathrm{GAG}>\mathrm{AAG}$ & R110C, Q100stop, P36L & NA \\
\hline MAGEA1 & 6227 & D258A & $\mathrm{GAT}>\mathrm{GCT}$ & FS at $\mathrm{H} 179^{*}$ & WT \\
\hline MAGEA1 & 6613 & R236K & $A G G>A A G$ & R181P & WT \\
\hline MAGEA4 & 6541 & G316R & $\mathrm{GGA}>\mathrm{AGA}$ & WT & V600E \\
\hline MAGEA4 & 7889 & S99L & TCG $>$ TTG & R282P & WT \\
\hline MAGEA4 & 4198 & E138K & $\mathrm{GAG}>\mathrm{AAG}$ & R110C, Q100stop, P36L & NA \\
\hline MAGEA4 & 4198 & P149S & $\mathrm{CCT}>\mathrm{TCT}$ & R110C, Q100stop, P36L & NA \\
\hline MAGEA4 & 6985 & E224K & $\mathrm{GAG}>\mathrm{AAG}$ & WT & WT \\
\hline MAGEA4 & 02-105 & P267S & $\mathrm{CCT}>\mathrm{TCT}$ & WT & WT \\
\hline MAGEA4 & 7194 & E242K & $\mathrm{GAG}>\mathrm{AAG}$ & R196stop & WT \\
\hline MAGEA4 & $03-043$ & $\mathrm{R} 269 \mathrm{C}$ & $\mathrm{CGC}>\mathrm{TGC}$ & WT & WT \\
\hline MAGEA4 & 5668 & $|222|$ & ATC $>$ ATT & WT & WT \\
\hline MAGEA4 & 2112 & E21E & $\mathrm{GAG}>\mathrm{GAA}$ & WT & V600E \\
\hline MAGEA4 & 5558 & P45S & $\mathrm{CCT}>\mathrm{TCT}$ & S241F & WT \\
\hline MAGEC1 & $07-223$ & F904F & $\pi \mathrm{TC}>\mathrm{TT}$ & WT & V600E \\
\hline MAGEC1 & 04-007 & P26L & $\mathrm{CCT}>\mathrm{CTT}$ & WT & V600E \\
\hline MAGEC1 & 04-007 & L35F & $\mathrm{CTC}>\mathrm{TTC}$ & WT & V600E \\
\hline MAGEC1 & 7889 & E991K & $\mathrm{GAG}>\mathrm{AAG}$ & R282P & WT \\
\hline MAGEC1 & 4198 & E59E & $\mathrm{GAG}>\mathrm{GAA}$ & R110C, Q100stop, P36L & NA \\
\hline MAGEC1 & 4198 & D62N & $\mathrm{GAC}>\mathrm{AAC}$ & R110C, Q100stop, P36L & NA \\
\hline MAGEC1 & 4198 & P38S & $\mathrm{CCC}>\mathrm{TCC}$ & R110C, Q100stop, P36L & NA \\
\hline MAGEC1 & 6985 & P83S & $\mathrm{CCC}>\mathrm{TCC}$ & WT & WT \\
\hline MAGEC1 & 6985 & S688F & $\mathrm{TCC}>\mathrm{TTC}$ & WT & WT \\
\hline MAGEC1 & 6985 & S863L & $\mathrm{TCA}>\mathrm{TTA}$ & WT & WT \\
\hline MAGEC1 & 6458 & K1104K & $A A G>A A A$ & R248W & WT \\
\hline MAGEC1 & 7151 & P119S & $\mathrm{CCT}>\mathrm{TCT}$ & WT & V600E \\
\hline MAGEC1 & 7194 & Q664stop & CAG $>$ TAG & R196stop & WT \\
\hline MAGEC1 & 7194 & E668E & $\mathrm{GAG}>\mathrm{GAA}$ & R196stop & WT \\
\hline MAGEC1 & 7194 & P127L & $\mathrm{CCT}>\mathrm{CTT}$ & R196stop & WT \\
\hline MAGEC1 & 4066 & F904F & $\mathrm{TTC}>\mathrm{TTT}$ & WT & V600E \\
\hline MAGEC1 & $02-024$ & L705L & $\mathrm{CTG}>\mathrm{TTG}$ & R213stop & WT \\
\hline MAGEC1 & 6795 & G986E & $\mathrm{GGG}>\mathrm{GAG}$ & WT & WT \\
\hline MAGEC1 & 4985 & S964S & $\mathrm{TCC}>\mathrm{TCA}$ & WT & WT \\
\hline MAGEC1 & 4985 & S18S & $\mathrm{TCC}>\mathrm{TCT}$ & WT & WT \\
\hline MAGEC1 & 4985 & D687N & GAT $>$ AAT & WT & WT \\
\hline MAGEC1 & 4062 & P50S & $\mathrm{CCT}>\mathrm{TCT}$ & WT & V600K \\
\hline MAGEC1 & $03-063$ & S134S & $\mathrm{TCC}>\mathrm{TCT}$ & WT & G596R \\
\hline MAGEC2 & $07-223$ & P3S & $\mathrm{CCC}>\mathrm{TCC}$ & WT & V600E \\
\hline MAGEC2 & 4198 & F151Y & $\mathrm{TTC}>\mathrm{TAC}$ & R110C, Q100stop, P36L & NA \\
\hline MAGEC2 & 4198 & E36E & $\mathrm{GAG}>\mathrm{GAA}$ & R110C, Q100stop, P36L & NA \\
\hline MAGEC2 & 6985 & S58F & $\mathrm{TCC}>\mathrm{TTC}$ & WT & WT \\
\hline MAGEC2 & 7516 & F265F & $\pi \mathrm{TC}>\mathrm{TT}$ & R337S & WT \\
\hline MAGEC2 & 7259 & D335N & GAT $>$ AAT & WT & WT \\
\hline MAGEC2 & $02-102$ & P84P & $\mathrm{CCC}>\mathrm{CCT}$ & WT & V600E \\
\hline MAGEE1 & 6541 & D446C & $\mathrm{GAT}>\mathrm{TGT}^{*}$ & WT & V600E \\
\hline MAGEE1 & 4198 & $\mathrm{R} 711 \mathrm{~K}$ & $A G G>A A G$ & R110C, Q100stop, P36L & NA \\
\hline MAGEE1 & 6985 & S319S & $\mathrm{TCC}>\mathrm{TCT}$ & WT & WT \\
\hline MAGEE1 & 03-091 & A717V & $\mathrm{GCT}>\mathrm{GTT}$ & WT & G469E \\
\hline
\end{tabular}


Table 2. Cont.

\begin{tabular}{llllll}
\hline Gene & Sample name & Amino acid & Codon change & TP53 status & BRAF status \\
\hline MAGEE1 & 08-249E & A859A & GCC $>$ GCT & WT & V600K \\
MAGEE1 & 8022 & S67F & TCC $>$ TTC & WT & V600E \\
MAGEE1 & 4985 & E692D & GAA $>$ GAT & WT & WT \\
MAGEE1 & 4985 & E693K & GAA $>$ AAA & WT & WT \\
\hline
\end{tabular}

doi:10.1371/journal.pone.0012773.t002

inactivating than activating. Consistent with this, for the MAGEA genes where the NS:S ratio is suggestive of their being drivers, there is evidence that both play tumor suppressive roles. MAGEA1 expression was shown to correlate with good prognosis in neuroblastoma [14] and MAGEA4 expression was shown to promote tumor cell death and sensitize lung malignancies to apoptotic stimuli, such as chemotherapeutic agents [16]. MAGEA4 was also shown to interact with gankyrin and to suppress its oncogenic activity [24]. It is thus possible, that the inactivation of MAGEA1 and MAGEA4, which are generally expressed in coordination with other CT-X genes such as MAGEA2 and $M A G E A 3$ for which there is evidence for oncogenic function, might enhance the overall tumorigenicity of coordinated CT-X expression leading to a net positive contribution to tumor progression.

Recently the X-ray structure of the MAGE homology domain of MAGEA4 (PDB ID 2WA0) was determined. This permitted a more detailed analysis of the mutations that fall within this domain in the various family members, as described in Supplementary Methods S1. We found that the mutations found in this region involve residues that have a higher solvent exposure and a lower FoldX score [25] than average, implying that they do not play an important role in the structural integrity of the protein but might serve for interactions with other molecules (Supplementary Methods S1 and Table S6). Although it is unclear whether the MAGE homology domain is involved in binding interactions, this observation reinforces the possibility of the mutations resulting in discrete functional changes.

One other aspect of the MAGE gene mutations worthy of further investigation is their frequent occurrence in potential phosphorylation sites. As predicted by http://scansite.mit.edu/, these mutations could either abolish predicted existing sites or create new potential sites (A63P [new site at S62], K278T and S296P in MAGEA1; P45S, S99L, P149S and P267S in MAGEA4; P38S, P83S and S86L in MAGEG1; S51F, S58F, S110N, S111L, F151Y and P295L [new sites at S293, Y296 and Y297] in

Table 3. Somatic mutations identified in MAGE genes in genome wide surveys.

\begin{tabular}{|c|c|c|c|c|c|c|}
\hline Gene & Sample name (tumor type) & Amino acid & Codon change & TP53 status & BRAF status & Reference \\
\hline MAGEA1 & HCC1008 (breast cancer cell line) & K278T & $\mathrm{AAA}>\mathrm{ACA}$ & $\mathrm{D} 281 \mathrm{H}$ & WT & [17] \\
\hline MAGEA1 & NCI-H1770 (NSCLC cell line) & A63P & $\mathrm{GCC}>\mathrm{CCC}$ & R248W & WT & [22] \\
\hline MAGEA4 & HCC1008 (breast cancer cell line) & G153D & $\mathrm{GGC}>\mathrm{GAC}$ & $\mathrm{D} 281 \mathrm{H}$ & WT & [17] \\
\hline MAGEA4 & Br27P (glioma) & E221K & $\mathrm{GAA}>\mathrm{AAA}$ & c.617delT (fs) & T310l & [18] \\
\hline MAGEB6B & NCI-H2087 (NSCLC cell line) & $\mathrm{G} 71>\mathrm{F}$ & $\mathrm{GGT}>\pi T$ & V157F & L597V & [22] \\
\hline MAGEB10 & Bro9PT (glioma) & D55Y & GAT $>$ TAT & W53X & WT & [18] \\
\hline MAGEB10 & LB647-SCLC (SCLC cell line) & Q148K & CAG $>$ AAG & p.E294fs*51 & WT & [22] \\
\hline MAGEB16 & NCI-H2009 (NSCLC cell line) & T302R & $A C A>A G A$ & R273L & WT & [22] \\
\hline MAGEB16 & LB647-SCLC (SCLC cell line) & A279T & $\mathrm{GCT}>\mathrm{ACT}$ & p.E294fs*51 & WT & [22] \\
\hline MAGEB16 & HCC2218 (breast cancer cell line) & L323L & $\mathrm{CTG}>\mathrm{CTT}$ & $\mathrm{R} 283 \mathrm{C}$ & WT & [17] \\
\hline MAGEC1 & Br02X (glioma) & $11001 \mathrm{~F}$ & $\mathrm{~A} \Pi \mathrm{T}>\mathrm{TT}$ & WT & WT & [18] \\
\hline MAGEC2 & HCC1954 (breast cancer cell line) & G6C & $\mathrm{GGC}>\mathrm{TGC}$ & $\mathrm{Y} 163 \mathrm{C}$ & WT & [17] \\
\hline MAGEC3 & MZ7-mel (melanoma cell line) & D50N & $\mathrm{GAC}>\mathrm{AAC}$ & WT & V600E & [22] \\
\hline MAGEC3 & CP66-MEL (melanoma cell line) & L551F & $\mathrm{CTT}>\mathrm{TT}$ & WT & WT & [22] \\
\hline MAGED2 & Hs 578T (breast cancer cell line) & K458Q & $A A G>C A G$ & V157F & WT & [22] \\
\hline MAGEE1 & HCC2713 (breast cancer cell line) & Y640F & $\mathrm{TAC}>\mathrm{TTC}$ & c.723delC (fs) & NA & [22] \\
\hline MAGEE 1 & CP66-MEL (melanoma cell line) & R934K & $A G G>A A G$ & WT & WT & [22] \\
\hline MAGEE1 & HCC1008 (breast cancer cell line) & T664N & $A C C>A A C$ & $\mathrm{D} 281 \mathrm{H}$ & WT & [17] \\
\hline MAGEE1 & Pa14C (pancreas tumor) & V649V & $\mathrm{GTG}>\mathrm{GTT}$ & WT & WT & [19] \\
\hline MAGEH1 & $\mathrm{Br} 23 \mathrm{X}$ (glioma) & A13A & $\mathrm{GCG}>\mathrm{GCA}$ & WT & WT & [18] \\
\hline MAGEH1 & NCI-H2087 (NSCLC cell line) & F100F & $\pi \mathrm{TC}>\pi T$ & V157F & L597V & [22] \\
\hline
\end{tabular}

*Two consecutive changes.

doi:10.1371/journal.pone.0012773.t003 

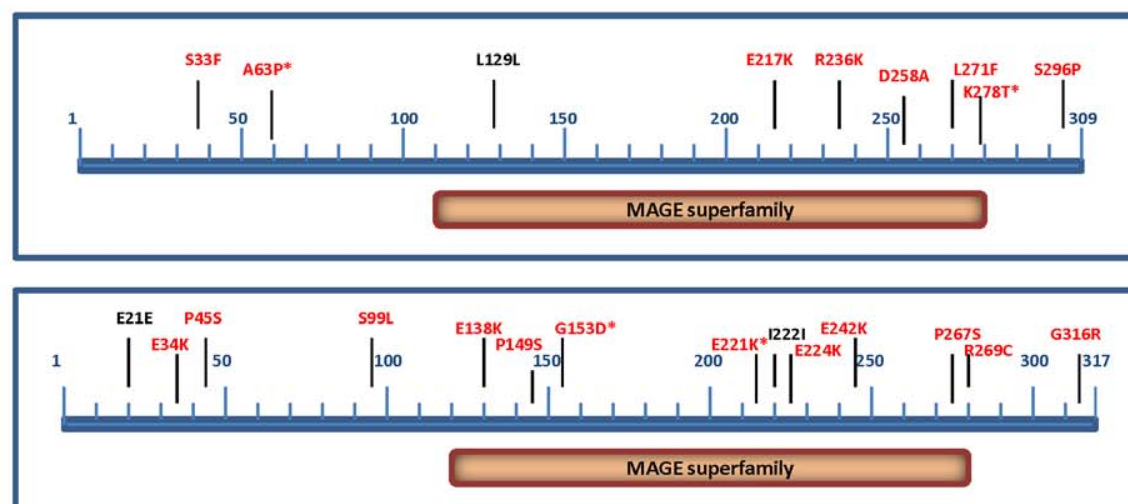

MAGEA4

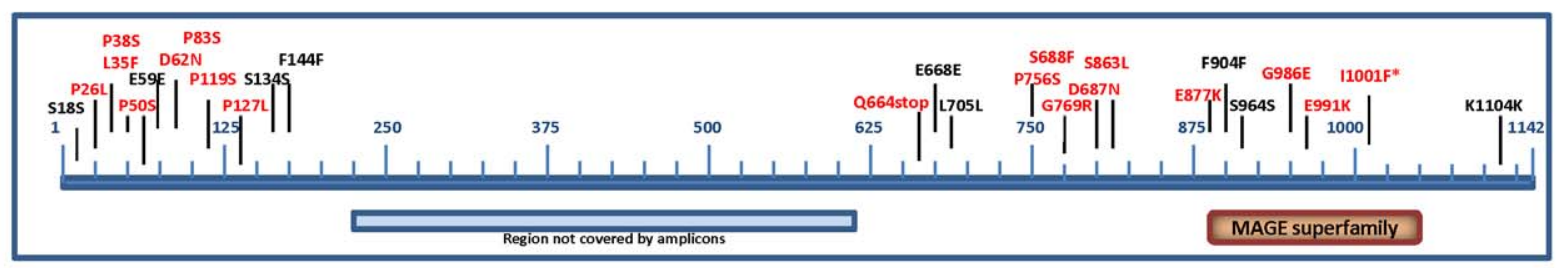

MAGEC1

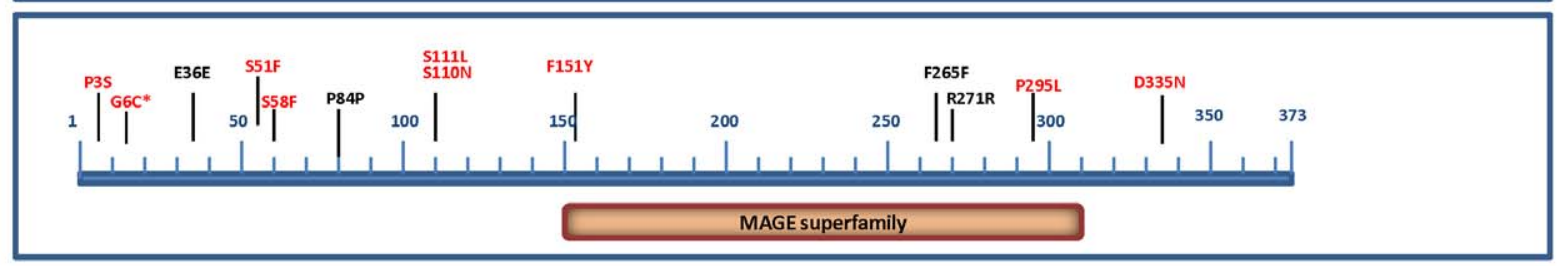

MAGEC2

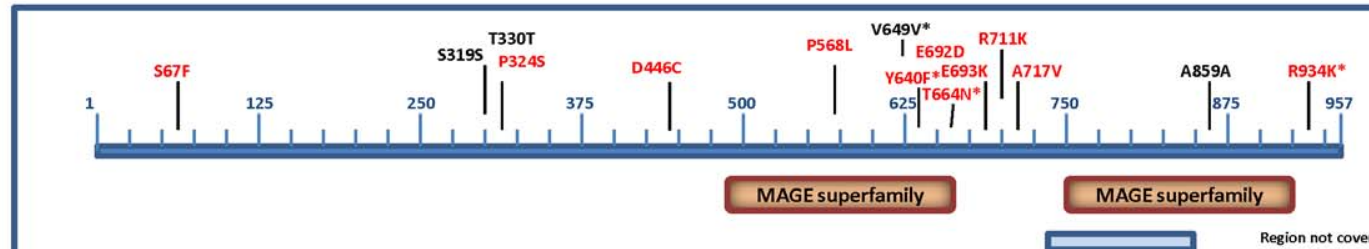

MAGEE1 mutations. Asterisks indicate mutations that were identified in other studies. doi:10.1371/journal.pone.0012773.g001

MAGEC2). Although at this stage we have no direct evidence that the MAGE proteins are phosphorylated, this observation does hint at potential functional consequence of many of the mutations.

Lastly, we considered the possibility that selective immune pressure that might underlie the MAGE mutations. In this context, six of the eight missense mutations identified in MAGEA1 (residues $63,236,258,271,278,296)$, the only one of the genes sequenced where extensive mapping of $\mathrm{T}$ cell epitopes has been performed, affect known epitopes [26]. Thus, the mutations might reduce antigenicity and serve as an alternate escape mechanism to loss of antigen or MHC expression. It remains to be determined, however, whether the mutated epitopes were involved in antigenicity in the patients where they were identified.

A notable facet of the MAGE mutations is their non-random distribution between patients. Thirty five of the fifty four $M A G E$ mutations $(64.8 \%)$ in our Validation and Discovery Screens are from samples that exhibit more than one mutation, often with multiple mutations in the same gene. For example, one sample in the Validation Screen, 4198, exhibited nine coding region mutations and another, 6985, six mutations. Even in the list of mutations identified in other studies, $41 \%$ are from samples where more than one $M A G E$ mutation has been identified. These data suggest that a significant subset of the mutations might arise due to a DNA instability syndrome, either affecting the X-chromosome or the entire genome.

While our results do not as yet define the functional consequences of the MAGE gene mutations observed, they do demonstrate for the first time that this gene family is frequently mutated in melanoma. Therefore, our study argues for enhanced efforts to discern potential tumorigenic properties of these genes that serve as the platform for therapeutic cancer vaccines already in advanced clinical development.

\section{Supporting Information}

Supplementary Methods S1 Homology Models of the MAGE Homology Domain

Found at: doi:10.1371/journal.pone.0012773.s001 (0.05 MB DOC)

Table S1 Melanoma cell lines used in the Discovery Screen Found at: doi:10.1371/journal.pone.0012773.s002 (0.03 MB XLS)

Table S2 Clinical characteristics of the Melanoma patients included in the Validation Screen 
Found at: doi:10.1371/journal.pone.0012773.s003 (0.13 MB XLS)

Table S3 Primer sequences used in this study Found at: doi:10.1371/journal.pone.0012773.s004 (0.06 MB XLS)

Table S4 Somatic TP53 mutations identified in ovarian tumors Found at: doi:10.1371/journal.pone.0012773.s005 (0.03 MB DOC)

Table S5 Spectrum of the MAGE mutations in the discovery and validation sets

Found at: doi:10.1371/journal.pone.0012773.s006 (0.04 MB DOC)

\section{References}

1. Simpson AJ, Caballero OL, Jungbluth A, Chen YT, Old LJ (2005) Cancer/testis antigens, gametogenesis and cancer. Nat Rev Cancer 5: 615-625.

2. De Plaen E, Arden K, Traversari C, Gaforio JJ, Szikora JP, et al. (1994) Structure, chromosomal localization, and expression of 12 genes of the MAGE family. Immunogenetics 40: 360-369.

3. Caballero OL, Chen YT (2009) Cancer/testis (CT) antigens: potential targets for immunotherapy. Cancer Sci 100: 2014-2021.

4. van der Bruggen P, Traversari C, Chomez P, Lurquin C, De Plaen E, et al. (1991) A gene encoding an antigen recognized by cytolytic T lymphocytes on a human melanoma. Science 254: 1643-1647.

5. Brichard VG, Lejeune D (2008) Cancer immunotherapy targeting tumourspecific antigens: towards a new therapy for minimal residual disease. Expert Opin Biol Ther 8: 951-968.

6. Yang B, O'Herrin SM, Wu J, Reagan-Shaw S, Ma Y, et al. (2007) MAGE-A, mMage-b, and MAGE-C proteins form complexes with KAP1 and suppress p53-dependent apoptosis in MAGE-positive cell lines. Cancer Res 67: 9954-9962.

7. Monte M, Simonatto M, Peche LY, Bublik DR, Gobessi S, et al. (2006) MAGEA tumor antigens target p53 transactivation function through histone deacetylase recruitment and confer resistance to chemotherapeutic agents. Proc Natl Acad Sci U S A 103: 11160-11165.

8. Atanackovic D, Hildebrandt Y, Jadczak A, Cao Y, Luetkens T, et al. (2010) Cancer-testis antigens MAGE-C1/CT7 and MAGE-A3 promote the survival of multiple myeloma cells. Haematologica 95: 785-793.

9. Liu W, Cheng S, Asa SL, Ezzat S (2008) The melanoma-associated antigen A3 mediates fibronectin-controlled cancer progression and metastasis. Cancer Res 68: 8104-8112.

10. Jeon CH, Shin IH, Park JB, Chae HD (2010) Prognostic Significance of MAGE in Peritoneal Washes in Gastric Carcinoma Patients Without Peritoneal Metastasis: Results of a 5-year Follow-up Study. J Clin Gastroenterol.

11. Gure AO, Chua R, Williamson B, Gonen M, Ferrera CA, et al. (2005) Cancertestis genes are coordinately expressed and are markers of poor outcome in nonsmall cell lung cancer. Clin Cancer Res 11: 8055-8062.

12. Atanackovic D, Luetkens T, Hildebrandt Y, Arfsten J, Bartels K, et al. (2009) Longitudinal analysis and prognostic effect of cancer-testis antigen expression in multiple myeloma. Clin Cancer Res 15: 1343-1352.
Table S6 Mutations in MAGE proteins, FoldX score, solvent accessible surface area and conservation score.

Found at: doi:10.1371/journal.pone.0012773.s007 (0.04 MB DOC)

\section{Acknowledgments}

We thank K. Muehlethaler and C. Beauverd for excellent technical help.

\section{Author Contributions}

Conceived and designed the experiments: OLG QZ RLS AJS. Performed the experiments: OLC QZ DR SS SD. Analyzed the data: OLC QZ DR BJS SD UFR AP OM BJVdE LJO XL JC RLS AJS. Contributed reagents/materials/analysis tools: DR BJS SS UFR AP OM DS JDW CL TP KO FB BJVdE LJO XL JC. Wrote the paper: OLG RLS AJS.

13. Bergeron A, Picard V, LaRue H, Harel F, Hovington H, et al. (2009) High frequency of MAGE-A4 and MAGE-A9 expression in high-risk bladder cancer. Int J Cancer 125: 1365-1371.

14. Grau E, Oltra S, Martinez F, Orellana C, Canete A, et al. (2009) MAGE-A1 expression is associated with good prognosis in neuroblastoma tumors. J Cancer Res Clin Oncol 135: 523-531.

15. Sharma P, Shen Y, Wen S, Bajorin DF, Reuter VE, et al. (2006) Cancer-testis antigens: expression and correlation with survival in human urothelial carcinoma. Clin Cancer Res 12: 5442-5447.

16. Peikert T, Specks U, Farver C, Erzurum SC, Comhair SA (2006) Melanoma antigen A4 is expressed in non-small cell lung cancers and promotes apoptosis. Cancer Res 66: 4693-4700

17. Wood LD, Parsons DW, Jones S, Lin J, Sjoblom T, et al. (2007) The genomic landscapes of human breast and colorectal cancers. Science 318: 1108-1113.

18. Parsons DW, Jones S, Zhang X, Lin JC, Leary RJ, et al. (2008) An integrated genomic analysis of human glioblastoma multiforme. Science 321: 1807-1812.

19. Jones S, Zhang X, Parsons DW, Lin JC, Leary RJ, et al. (2008) Core signaling pathways in human pancreatic cancers revealed by global genomic analyses. Science 321: 1801-1806.

20. Pejovic T, Pande NT, Mori M, Mhawech-Fauceglia P, Harrington C, et al. (2009) Expression profiling of the ovarian surface kinome reveals candidate genes for early neoplastic changes. Transl Oncol 2: 341-349.

21. Singer G, Stohr R, Cope L, Dehari R, Hartmann A, et al. (2005) Patterns of p53 mutations separate ovarian serous borderline tumors and low- and high-grade carcinomas and provide support for a new model of ovarian carcinogenesis: a mutational analysis with immunohistochemical correlation. Am J Surg Pathol 29: 218-224.

22. COSMIC (Catalogue Of Somatic Mutations In Cancer) (accessed 2010) http:// www.sanger.ac.uk/genetics/CGP/cosmic/.

23. Pleasance ED, Cheetham RK, Stephens PJ, McBride DJ, Humphray SJ, et al. (2010) A comprehensive catalogue of somatic mutations from a human cancer genome. Nature 463: 191-196.

24. Nagao T, Higashitsuji H, Nonoguchi K, Sakurai T, Dawson S, et al. (2003) MAGE-A4 interacts with the liver oncoprotein gankyrin and suppresses its tumorigenic activity. J Biol Chem 278: 10668-10674.

25. Guerois R, Nielsen JE, Serrano L (2002) Predicting changes in the stability of proteins and protein complexes: a study of more than 1000 mutations. J Mol Biol 320: 369-387. 